\title{
In-Situ Pulsed Laser Interference Nanostructuring of Semiconductor Surfaces
}

\author{
Yun-Ran Wang ${ }^{1}$, Im Sik Han ${ }^{1}$, Chao-Yuan Jin ${ }^{1,2}$, and Mark Hopkinson ${ }^{* 1}$ \\ ${ }^{1}$ Department of Electronic and Electrical Engineering, University of Sheffield, Sheffield, S3 7HQ, \\ United Kingdom \\ ${ }^{2}$ College of Information Science and Electronic Engineering, Zhejiang University, Hangzhou \\ 310007, China \\ *Corresponding author's e-mail: m.hopkinson@sheffield.ac.uk
}

\begin{abstract}
We report the fabrication of periodic one- and two-dimensional nanostructures on semiconductor surfaces utilizing in-situ single-pulse direct laser interference patterning during molecular beam epitaxial growth. A Nd:YAG laser operating at $355 \mathrm{~nm}$ wavelength with a pulse duration of $7 \mathrm{~ns}$ is used to produce periodic gratings or nanoislands with a submicron periodicity on the growing InAs/GaAs surfaces. By means of four-beam interference patterning, ordered square arrays of quantum dots with a lattice pitch of 200-300 nm are obtained. This in-situ technique offers a new way to control the quantum dot nucleation sites and it constitutes a technological advance to realize periodic III-V semiconductor nanostructures with impact in optoelectronics and quantum information processing.

DOI: $10.2961 /$ jlmn.2020.02.2011
\end{abstract}

Keywords: direct laser interference patterning, multi-beam interference, nanostructures, III-V semiconductors, quantum dots, molecular beam epitaxy

\section{Introduction}

The development of micro- and nanofabrication technologies is at the research frontline of the semiconductor device revolution. Periodic semiconductor structures with feature sizes as small as hundreds of nanometers exhibit peculiar optical and electronic properties and have attracted increasing interests in the fields of photonics, optoelectronics, and bioengineering [1-3]. For example, nanopatterns on solar cells and light emitting diodes show excellent antireflection property and enhanced optical performance [4,5]. Site-controlled low-dimensional semiconductor nanostructures such as quantum dots (QDs) and nanowires (NWs) has emerged as importance candidates for novel photonic devices and quantum information technologies [6-8]. Specifically, single quantum dot arrays are highly desirable for the integration of individual QDs into photonic cavities or other quantum circuits. Densely ordered arrays of QDs with periods of few hundred nanometers have shown great potential for longrange quantum correlations in quantum computation [9], and high density three-dimensional QD arrays are ideal for QD lasers [10].

Techniques such as electron-beam lithography [11] and focused ion-beam lithography [12] followed by chemical etching have been commonly implemented to fabricate semiconductor nanostructures; however, their relatively highcost equipment, the need for multiple processing steps and low throughput limit the application of industrial mass fabrication. Furthermore, as these are ex-situ methods, defects or contamination at the atomic scale could be introduced because of the multiple processing steps. Over the last decades, laser-based optical methods including direct laser writing (DLW) [13,14], laser interference lithography (LIL) $[15,16]$ and direct laser interference patterning (DLIP) [17-19] have been demonstrated to be attractive technologies for fast, cost-effective and high-throughput realization of one-, twoor three-dimensional periodic micro/nanostructures. Among them, DLIP utilizes the interference of two or more laser beams, presenting a parallel, photoresist-free structuring approach which allows one to directly pattern the sample surfaces in a single processing step. Many excellent works show that DLIP is capable of patterning a wide variety of materials including metals, ceramics, polymers and semiconductors [18-23], and more recently it has shown great potential in the epitaxial growth and fabrication of III-V semiconductor nanostructures [24-27].

In this paper, we demonstrate an in-situ DLIP approach to structure epitaxial semiconductors grown by molecular beam epitaxy (MBE). Periodic one-dimensional (1D) gratings and two-dimensional (2D) nanoislands can be created, and on the basis of nanoisland arrays, we are able to fabricate highly ordered arrays of InAs QDs. These results show that single-pulse DLIP is a powerful technique for the direct, fast and high-throughput fabrication of semiconductor nanostructures.

\section{Theoretical analysis and simulations}

Multi-beam interference can be described as a superposition of coherent light waves. The intensity distribution can be described as the superposition of the electric field vectors. The electric field vector of the $m^{\text {th }}$ beam can be expressed as

$$
\overrightarrow{E_{m}}=A_{m} \overrightarrow{P_{m}} \exp \left[i\left(\overrightarrow{k_{m}} \cdot \vec{r}+\delta_{m}\right)\right] \text { (1) }
$$

where $A_{m}$ is the amplitude, $\vec{P}_{m}$ is the unit polarization vector, $\overrightarrow{k_{m}}$ is the wave vector, $\vec{r}$ is the position vector, and $\delta_{m}$ is the initial phase. In Eq. (1) $\overrightarrow{P_{m}}, \overrightarrow{k_{m}}$ and $\vec{r}$ can be written as 


$$
\begin{gathered}
\overrightarrow{P_{m}}=-\left(\cos \theta_{m} \cos \varphi_{m} \cos \psi_{m}-\sin \varphi_{m} \sin \psi_{m}\right) \cdot \vec{i} \\
-\left(\cos \theta_{m} \sin \varphi_{m} \cos \psi_{m}+\cos \varphi_{m} \sin \psi_{m}\right) \cdot \vec{j} \\
-\left(\sin \theta_{m} \cos \psi_{m}\right) \cdot \vec{k} \\
\overrightarrow{k_{m}}=k\left(\sin \theta_{m} \cos \varphi_{m} \cdot \vec{i}+\sin \theta_{m} \sin \varphi_{m} \cdot \vec{j}-\cos \theta_{m} \cdot \vec{k}\right) \\
\vec{r}=x \cdot \vec{i}+y \cdot \vec{j}+z \cdot \vec{k}
\end{gathered}
$$

where $k=2 \pi / \lambda$ is the wave number, $\lambda$ is the wavelength of the laser, $\theta_{m}$ represents the angle of incidence, $\varphi_{m}$ is the azimuthal angle, $\psi_{m}$ is the polarization angle. The intensity distribution of N-beam interference field in the $\mathrm{z}=0$ plane can be calculated by

$$
I=\sum_{n=1}^{N} \sum_{m=1}^{N} A_{n} A_{m} \overrightarrow{P_{n}} \vec{P}_{m} \exp \left\{i\left[\left(\overrightarrow{k_{n}}-\overrightarrow{k_{m}}\right) \cdot \vec{r}+\left(\delta_{n}-\delta_{m}\right)\right]\right\}
$$
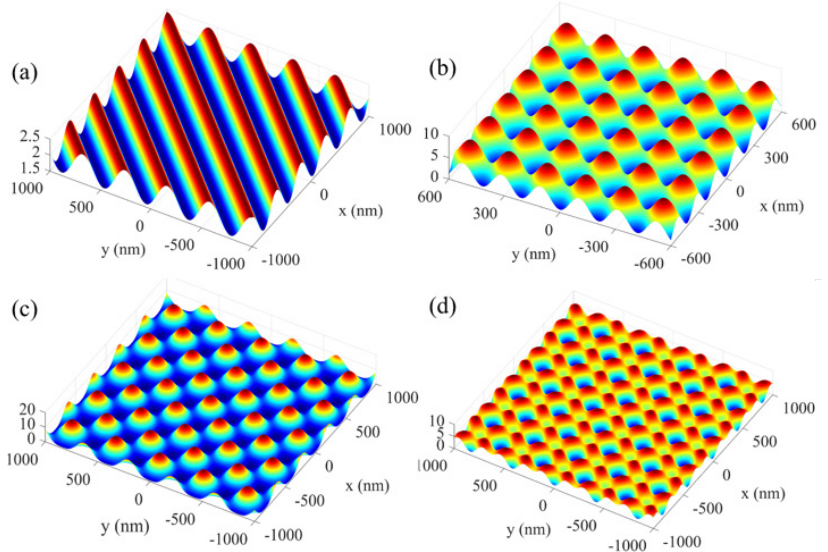

Fig. $13 D$ MATLAB simulation results of (a) two-beam interference with azimuth angles of $\varphi_{1}=0^{\circ}$ and $\varphi_{2}=90^{\circ}$, and polarization angle of $0^{\circ}$, and four-beam interference with different polarization angles of (b) $90^{\circ}$ (TE), (c) $0^{\circ}$ (TM) and (d) $58^{\circ}$.

The simulated two-beam and four-beam interference patterns are shown in Fig. 1, using the laser wavelength of 355 $\mathrm{nm}$, the incident angle of $58^{\circ}$, and the azimuthal angles of $\varphi_{1}=0^{\circ}, \varphi_{2}=90^{\circ}, \varphi_{3}=180^{\circ}, \varphi_{4}=270^{\circ}$. The amplitudes of all beams are assumed to be identical and the initial phases are all zero. The period of the interference pattern is decided by the wavelength of the laser beam $\lambda$ and the angle of incidence $\theta$, which gives $\Lambda=\lambda / \sqrt{2} \sin \theta$. In case of TE polarization mode (Fig. 1(b)), the period $\Lambda=\lambda / 2 \sin \theta$. By adopting these parameters an interference period of around $300 \mathrm{~nm}$ can be produced, which is desirable for the fabrication of state-ofthe-art periodic semiconductor nanostructures.

\section{Experimental}

\subsection{Integration of laser interference with $\mathrm{MBE}$}

The molecular beam epitaxy system (MBEKomponenten Octoplus 600) used is a typical small vertical geometry chamber. It has four symmetric anti-reflective optical vacuum viewports facing upwards from the lower half of the MBE system towards the substrate at an angle of incidence of $58^{\circ}$ for laser interference. Using such an arrangement, a lattice pitch $\Lambda=209 \mathrm{~nm}$ for the TE polarization state and $\Lambda=296 \mathrm{~nm}$ for other polarization states can be achieved.

The overall optical arrangement, without the chamber, is shown in Fig. 2(a). Laser interference was realized using a flash-lamp pumped Nd:YAG laser (Innolas Spitlight), operating at a wavelength of $355 \mathrm{~nm}\left(\mathrm{M}^{2}<1.3\right)$, generated from a third harmonic from a $1064 \mathrm{~nm}$ laser, with a pulse duration of $7 \mathrm{~ns}$. The beam diameter on the sample surface is around $5 \mathrm{~mm}$ with a gaussian intensity distribution. The output laser beam was split into four beams by three dichroic beam splitters. The polarization state and laser energy of each beams were controlled by means of a half waveplate and a Glanlaser polarizer. An external shutter was used to pick a single pulse from the $5 \mathrm{~Hz}$ pulse train for the experiments. It is important to note that although the laser system can provide a single pulse, the stability of the pulse energy requires thermal equilibrium to be achieved, which is not established for at least a few hundred pulses. It is therefore necessary to run the laser in a continuous pulsed mode and select pulses when stability is reached, which typically takes $\sim 1-2$ mins.

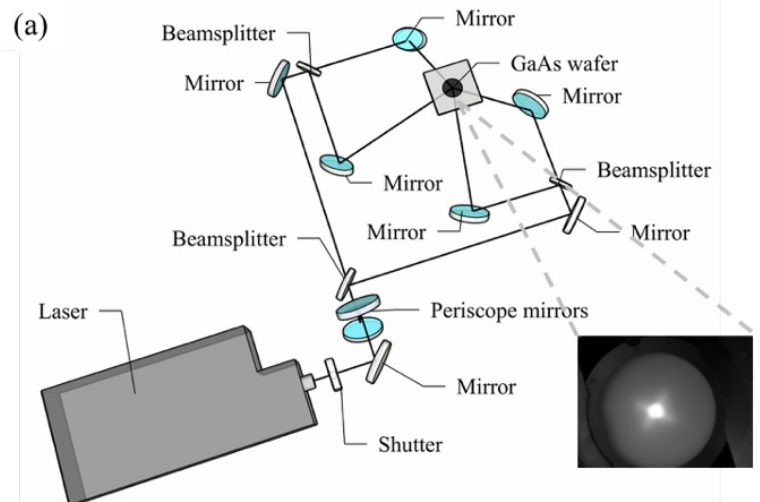

(b)

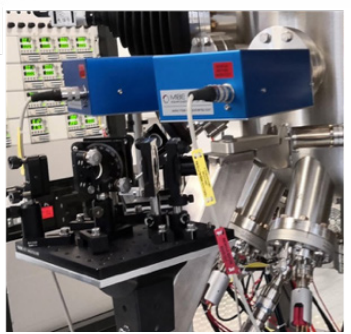

(c)

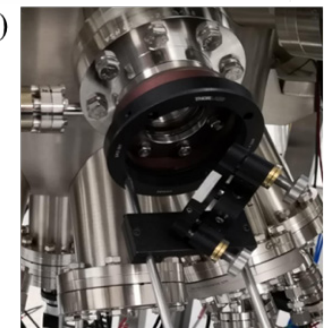

Fig. 2 (a) Schematic configuration of the four-beam DLIP-MBE setup. The insect is an image of the four superimposed beams on the surface of an InGaN test wafer. (b) Optics platform with optical components attached to the MBE system sub-frame. (c) A beam steering mirror attached to the optical viewport.

Optical components such as mirrors and beam splitters are built up on platforms (see Fig. 2(b)) which are attached to the MBE sub-frame, whilst the light is guided into the MBE system through viewports by means of mirrors attached to the ports (see Fig. 2(c)). The final alignment process involves guiding four beams to the centre of a 2 -inch wafer held on the substrate stage of the MBE system and pointing downwards. For the purposes of viewing the UV beams we were able to observe blue luminescence from an InGaN/GaN quantum well wafer with a Si CMOS camera.

\subsection{MBE growth patterning}

All the samples were grown on 2-inch epi-ready GaAs (100) wafers by MBE. Following native oxide desorption, a $500 \mathrm{~nm}$-thick GaAs buffer layer at a substrate temperature of $600{ }^{\circ} \mathrm{C}$ was grown, at the end of which the substrate temperature was lowered to $500{ }^{\circ} \mathrm{C}$. The resulting buffer layers generally show wide monolayer terraces with a high degree of atomic flatness. Deposition of around 1.6-1.8 monolayers (ML) of InAs on this surface is sufficient to nucleate InAs 


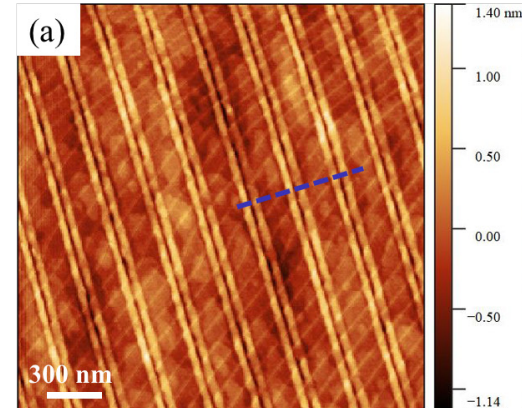

(d)

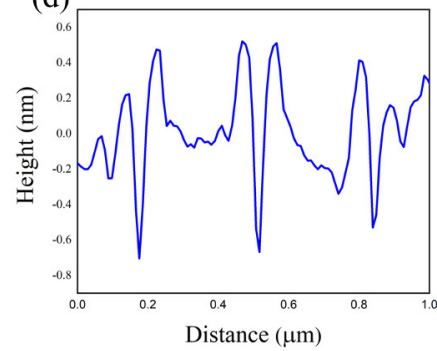

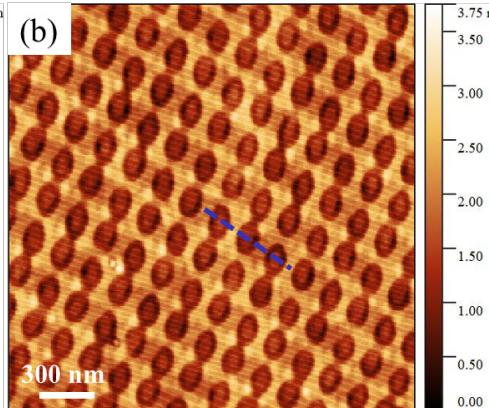

(e)

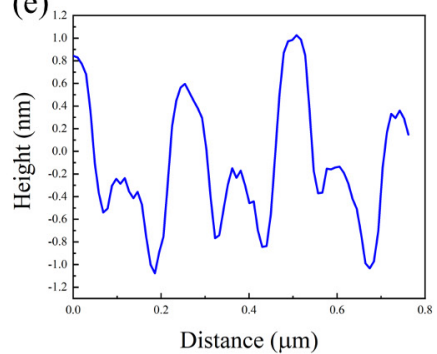

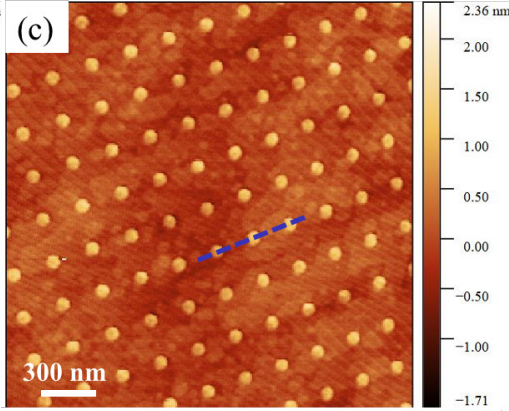

(f)

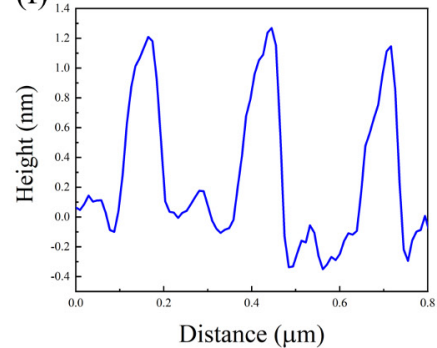

Fig. $33 \times 3 \mu \mathrm{m}^{2}$ AFM images of fabricated periodic nanostructures on 1 ML InAs layers patterned by (a) two-beam interference and fourbeam interference with polarization angles of (b) $90^{\circ}$ and (c) $58^{\circ}$. (d-f) The corresponding line profiles of these structures along the directions as marked with blue dotted line in (a-c).

QDs. Without patterning, we observe the random nucleation of QDs with a density $\sim 100$ dots $/ \mu \mathrm{m}^{2}$.

For the DLIP approach, single-pulse direct laser interference patterning was applied with a UV laser fluence in the range of $10-30 \mathrm{~mJ} / \mathrm{cm}^{2}$ immediately after the deposition of a $1 \mathrm{ML}$ InAs layer. The InAs growth rate is $0.079 \mathrm{ML} / \mathrm{s}$ throughout. We have chosen $1 \mathrm{ML}$ since this is below the $2 \mathrm{D}$ layer to $3 \mathrm{D}$ dot transition and the InAs layer would be expected to remain relatively flat. Then, depending on the particular experiment, a further 0.6 ML may be grown on top of the patterned layer to induce QD nucleation. Following the InAs deposition the samples were quenched and taken out from the MBE chamber for surface structural characterization by atomic force microscopy (AFM). The patterned area extends over a region $\sim 3 \mathrm{~mm}$ diameter at the centre of the wafer where the four projected beams overlap.

\section{Results and discussions}

For samples with 1ML InAs, following single pulse patterning various surface features can be obtained. An AFM image of the fabricated periodic line-like structures with a period of around $300 \mathrm{~nm}$ is shown in Fig. 3(a), and its corresponding line profile is provided in Fig. 3(d). Two-beam interference with the configuration parameters presented in Fig. 1(a) was used with a laser fluence approximately 25 $\mathrm{mJ} / \mathrm{cm}^{2}$. At the interference maxima areas, one can clearly observe deep (black) lines with an average depth of $0.7 \mathrm{~nm}$ ( $\sim 2 \mathrm{ML})$ and besides these lines we can see the formation of about 1 ML-high mounds, which we attribute to be the migration of materials from the interference maxima. The patterns shown in Fig. 3(b) and (c) were generated from fourbeam interference with different polarization states, for which the utilized laser fluence was $31 \mathrm{~mJ} / \mathrm{cm}^{2}$ and 15 $\mathrm{mJ} / \mathrm{cm}^{2}$, respectively. The polarization state will affect the reflectance of the light on the GaAs surface and the interference intensity contrast as revealed in the simulation results. Fig. 3(b) demonstrates hole-like structures with a feature pe- riod as small as $210 \mathrm{~nm}$, which is consistent with the simulation result shown Fig. 1(b). The holes are relatively shallow around 2-3 ML depth. By changing the polarization direction, nanoisland-like structures with a period of $300 \mathrm{~nm}$ can be produced as presented in Fig. 3(c). The average height and diameter of these islands is $\sim 1 \mathrm{~nm}$ (3-4 ML) and $\sim 80 \mathrm{~nm}$, respectively. The formation of nanoislands we believe is owing to the accumulation of migrating atoms driven by thermal gradients under the influence of four-beam interference. A detailed description of the laser-induced adatom surface diffusion mechanism can be found in previous work $[26,28]$. Surface atoms can be able to move from interference maxima "hot" regions to interference minima "cold" regions. Consequently, the nanoislands will be formed in the "cold" regions.

The formation of periodic nanoislands after the singlepulse DLIP is capable of controlling the nucleation sites of InAs QDs. As can be seen in Fig. 4(a), after applying more InAs, small QDs start to nucleate at edges of the nanoislands. By depositing the InAs amount to around 1.75 ML, as displayed in Fig. 4(b), more QDs occur around the nanoislands. Therefore, the existence of nanoislands provides the preferential nucleation sites for QDs. By optimizing the growth conditions, such as to reduce the InAs coverage to 1.5-1.6 ML or decrease the InAs growth rate, InAs QD arrays with single or double dots per site can be obtained with a period of $300 \mathrm{~nm}$, as shown in Fig. 4(c) and (e). The area of the fabricated periodic QD arrays can reach several hundreds of square micrometers. Similarly, as presented in Fig. 4(d), QD arrays with a period of about $200 \mathrm{~nm}$ can also be produced by changing the laser polarization direction to TE and the InAs coverage here was $\sim 1.65$ ML. For comparison, Fig. 4(f) shows an AFM image of randomly nucleated InAs QDs with the InAs coverage of 1.6 ML, which is typical of epitaxially self-assembled QDs without pre-patterning. These results indicate that a single nanosecond laser pulse with relatively low fluence is able to control the growth of semiconductor 
nanostructures and can be very effective for laterally ordering the QDs. It is also important to note that in-situ DLIP is direct and contamination-free technique that does not require further chemical processing steps and enables a rapid fabrication with a single laser shot.
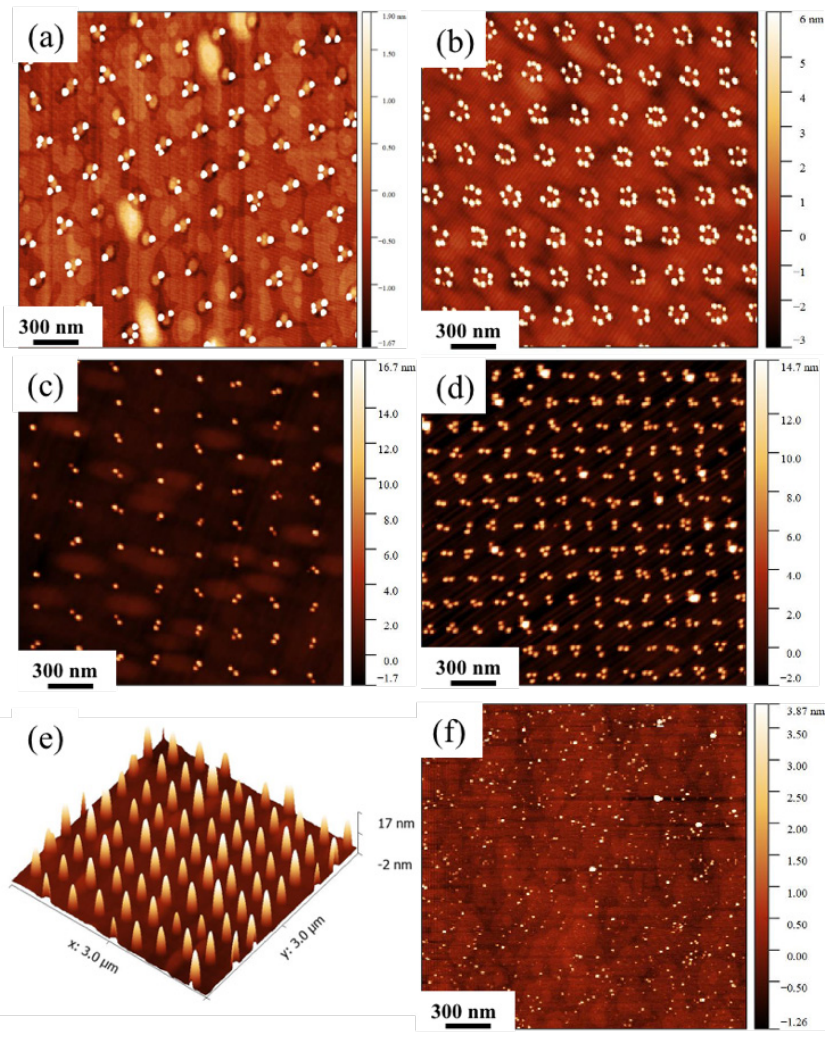

Fig. $43 \times 3 \mu \mathrm{m}^{2}$ AFM images of (a, b) InAs QDs nucleate at edges of nanoislands. The fabricated QD arrays with a period of (c) 300 $\mathrm{nm}$ and (d) $200 \mathrm{~nm}$. (e) A 3D AFM image of the optimized single dot arrays. (f) Non-patterned region of randomly distributed InAs QDs.

\section{Conclusion}

In this study, in-situ single-pulse direct laser interference patterning has been shown to be a fast and effective approach for the direct fabrication of periodic arrays of semiconductor surface nanostructures. Various structures were obtained including 1D gratings and 2D nanoislands by manipulating the interference parameters. Square arrays of InAs quantum dots with a period of $200 \mathrm{~nm}$ or $300 \mathrm{~nm}$ were achieved on GaAs substrates. This direct and high throughput in-situ technique provides a simple way of fabricating sub-micron resolution semiconductor nanostructures that is highly compatible with vacuum based epitaxial growth.

\section{Acknowledgments}

The authors gratefully acknowledge the support from the EU H2020 Program 767285 "Nanostencil”" and EPSRC.

\section{References}

[1] F. Priolo, T. Gregorkiewicz, M. Galli, and T.F. Krauss: Nat. Nanotechnol., 9, (2014) 19.

[2] S. Leung, Q. Zhang, F. Xiu, D. Yu, J. C. Ho, D. Li and Z. Fan: J. Phys. Chem. Lett., 5, (2014) 1479.

[3] Q. Luo, C. Hou, Y. Bai, R. Wang, and J. Liu: Chem. Rev., 116, (2016) 13571.
[4] J. Tippens, A. Bagal, X.A. Zhang, and C.H. Chang: Opt. Express, 25, (2017) A840.

[5] J.T. Chen, W.C. Lai, Y.J. Kao, Y.Y. Yang, and J.K. Sheu: Opt. Express, 20, (2012) 5689.

[6] J.O'Brien, A. Furusawa, J. Vuckovic: Nat. Photonics, 3, (2009) 687.

[7] P. Michler, A. Kiraz, C. Becher, W.V. Schoenfeld, P.M. Petroff, L. Zhang, E. Hu: Science, 290, (2000) 2282.

[8] Y.R. Wang, I.S. Han, C.Y. Jin, and M. Hopkinson: Appl. Phys. Lett., 116, (2020) 201901.

[9] F.R. Braakman, P. Barthelemy, C. Reichl, W. Wegscheider, and L.M. Vandersypen: Nat. Nanotechnol., 8, (2013) 432.

[10] D. Bimberg, M. Grundmann, F. Heinrichsdorff, N.N. Ledentsov, V.M. Ustinov, A.E. Zhukov, and A.F. Tsatsul'nikov: Thin Solid Films, 367, (2000) 235.

[11] C. Vieu, F. Carcenac, A. Pepin, Y. Chen, M. Mejias, A. Lebib, and H. Launois: Appl. Surf. Sci., 164, (2000) 111.

[12]F. Watt, A.A. Bettiol, J.A. Van Kan, E.J. Teo, and M.B.H. Breese: Int. J. Nanosci., 4, (2005) 269.

[13] M. Deubel, G.Von Freymann, M. Wegener, S. Pereira, K. Busch, and C.M. Soukoulis: Nat. Mater., 3, (2004) 444.

[14] M.S. Rill, C. Plet, M. Thiel, I. Staude, G.Von Freymann, S. Linden, and M. Wegener: Nat. Mater., 7, (2008) 543.

[15] A. Rodriguez, M. Echeverría, M. Ellman, N. Perez, Y.K. Verevkin, C.S. Peng, and S.M. Olaizola: Microelectron. Eng., 86, (2009) 937.

[16] C. Lu, and R.H. Lipson: Laser Photonics Rev., 4, (2010) 568.

[17] M. Bieda, M. Siebold, and A.F. Lasagni, Appl. Surf. Sci., 387, (2016) 175.

[18] A.F. Lasagni, D.F. Acevedo, C.A. Barbero, and F. Mücklich: Adv. Eng. Mater., 9, (2007) 99.

[19] M. Bieda, E. Beyer, A.F. Lasagni: J. Eng. Mater. Technol., 132 (2010) 031015.

[20] S. Alamri, and A.F. Lasagni: Opt. Express, 25, (2017) 9603.

[21] J. Berger, M.G. Holthaus, N. Pistillo, T. Roch, K. Rezwan, and A.F. Lasagni: Appl. Surf. Sci., 257, (2011) 3081 .

[22] C. Tan, C.S. Peng, J. Pakarinen, M. Pessa, V.N. Petryakov, Y.K. Verevkin, and S. Tisserand: Nanotechnology, 20, (2009) 125303.

[23] D. Wang, Z. Wang, Z. Zhang, Y. Yue, D. Li, and C. Maple: Appl. Surf. Sci., 282, (2013) 67.

[24] C.M. Clegg, and H. Yang: Sol. Energy Mater. Sol. Cells, 108, (2013) 252.

[25] W. Zhang, Z. Shi, D. Huo, X. Guo, F. Zhang, L. Chen, and C. Peng, Appl. Phys. Lett. 112, (2018) 153108.

[26] Y.R. Wang, I.S. Han, C.Y. Jin, and M. Hopkinson: ACS Appl. Nano. Mater., 3, (2020) 4739.

[27] Y.R. Wang, I.S. Han, C.Y. Jin, and M. Hopkinson: Accepted for publication in Opt. Express, https://doi.org/10.1364/OE.397709

[28] Y.R. Wang, C.Y. Jin, C.H. Ho, S. Chen, H. Francis, and M. Hopkinson: IET Optoelectron., 13, (2018) 7.

(Received: July 14, 2020, Accepted: September 7, 2020) 